\title{
Epigenetic Deregulation in Human Primary Immunodeficiencies
}

\author{
Elena Campos-Sanchez,, ${ }^{1,4}$ Jorge Martínez-Cano,, ${ }^{1,4}$ Lucía del Pino Molina, 2,3 \\ Eduardo López-Granados, ${ }^{2,3, \star}$ and Cesar Cobaleda ${ }^{1, \star}$
}

Primary immunodeficiencies (PIDs) are immune disorders resulting from defects in genes involved in immune regulation, and manifesting as an increased susceptibility to infections, autoimmunity, and cancer. However, the molecular basis of some prevalent entities remains poorly understood. Epigenetic control is essential for immune functions, and epigenetic alterations have been identified in different PIDs, including syndromes such as immunodeficiency-centromeric-instability-facial-anomalies, Kabuki, or Wolf-Hirschhorn, among others. Although the epigenetic changes may differ among these PIDs, the reversibility of epigenetic modifications suggests that they might become potential therapeutic targets. Here, we review recent mechanistic advances in our understanding of epigenetic alterations associated with certain PIDs, propose that a fully epigenetically driven mechanism might underlie some PIDs, and discuss the possible prophylactic and therapeutic implications.

The Heterogeneous Nature of Human Immunodeficiencies Human primary immunodeficiencies (PIDs) constitute a varied group of more than 350 immune disorders that cause an increased susceptibility to infections, autoimmunity, and cancer, linked to significant morbidity and mortality [1]. PIDs can appear clinically isolated or as a component of more complex multiorganic syndromes (syndromic immunodeficiencies). Most PIDs are genetic in origin, and new genes affected in PIDs are being described in increasing numbers [2]. Genetically unraveled PIDs are caused by either spontaneous or hereditary mutations affecting proteins with key roles in the development or function of cells of the immune system. The molecular pathways altered by these mutations can be manifold, and the clinical spectrum of PIDs is heterogeneous, with pathological consequences that can affect both immune and non-immune systems, ranging from just a reduced response to pathogens to problems such as autoimmunity and cancer [3]. Since they mainly have a genetic origin, the only definitive treatment for severe PIDs is hematopoietic stem cell transplantation (HSCT, see Glossary), which is a successful procedure, but still not exempt from significant morbidity and mortality. Autologous infusion of gene therapycorrected precursors is another curative procedure in a growing number of cases. Otherwise, lifelong immunoglobulin $(\mathrm{lg})$ replacement therapy (periodical intravenous or subcutaneos administration of nonspecific plasma-derived antibodies from healthy donors) is the main treatment for many patients with PID [4], together with prophylactic use of antibiotics, immunosupressants for autoimmune and inflammatory complications, and, more exceptionally, immunomodulating agents, such as interferon (IFN)- $\gamma$ or other cytokines [5].

Although many different immune cell types can be affected in PIDs, more than half of patients with PID present with Ig deficiencies [1,2] and, in the clinic, almost $90 \%$ of these patients have

\section{Highlights}

PIDs are rare disorders resulting from impaired function(s) in the immune system. Most are caused by mutations affecting proteins with key roles in the development or function of cells of the immune system.

Being mostly diseases of genetic origin, it is difficult to cure PIDs, and lifelong immunoglobulin (Ig) replacement therapy (periodical intravenous or subcutaneos administration of nonspecific plasma-derived antibodies from healthy donors) is the only treatment for many patients with PIDs.

Alterations in epigenetic regulators (mainly controlling DNA methylation, histone modifications, or chromatin remodeling) are increasingly being described for PIDs. Since epigenetic marks, unlike DNA mutations, can be pharmacologically reprogrammed, an understanding of how aberrant epigenetic patterns are initiated and maintained in PIDs might help us devise approaches to restore normal immune function.

${ }^{1}$ Department of Cell Biology and Immunology, Centro de Biología Molecular Severo Ochoa (CBMSO), CSIC/UAM, Madrid 28049, Spain ${ }^{2}$ Clinical Immunology Department, Hospital Universitario, La Paz Institute of Biomedical Research, 28046, Madrid, Spain

${ }^{3}$ Lymphocyte Pathophysiology Group, La Paz Institute of Biomedical Research, 28046 Madrid, Spain ${ }^{4}$ These authors contributed equally to this work

${ }^{*}$ Correspondence:

elgranados@salud.madrid.org (E. López-Granados) and cesar.cobaleda@csic.es (C. Cobaleda). 
defects in B cell differentiation [3], with disorders such as congenital agammaglobulinemias, class switch-recombination (CSR) or hyper-IgM (HIGM) defects, genetically defined and undefined common variable immunodeficiency (CVID), and selective IgA deficiency (IgAD) or lgG subclass deficiency. B cell development (reviewed in $[6,7]$ ) is a tightly regulated process requiring the coordinated action of extrinsic signals (cytokines, ligands, antigens, and interacting $T$ and dendritic cells), intrinsic factors (epigenetic regulators, transcription factors, and DNA recombination-activating proteins) and basic cellular processes (cellular proliferation and DNA repair). Therefore, interference with any of these elements can result in defective $B$ cell function and compromised antibody production. In recent years, alterations in genes involved in the epigenetic control of cellular identity and function (Boxes 1 and 2 ; Figure 1, Key Figure) have been associated with several PIDs, including syndromes such as immunodeficiencycentromeric-instability-facial-anomalies 1 (ICF1), Kabuki, Wolf-Hirschhorn (WHS), Schimke Immuno-Osseous Dysplasia (SIOD), Wiedemann-Steiner (WS), CHARGE, Kleefstra, Roifman, and INO80 loss-of-function (Figure 1). By analogy, the well-established role of epigenetic alterations in other pathologies has become an active area of investigation, as in the case of cancer $[8,9]$, complementing the previous mutation-centric interpretation of malignancies. Furthermore, due to their largely reversible nature, epigenetic modifications are quickly becoming crucial putative therapeutic targets in cancer and other diseases [10]. Here, we review recent advances in our understanding of the various epigenetic molecular mechanisms that have been shown to have a role in the aforementioned human PIDs. We also discuss the concept that other PID-causing mutations not previously known to be associated with epigenetic modifications may also act as epigenetic modifiers and, consequently, deregulated epigenetics might constitute one of the causes contributing to certain molecularly undefined PIDs. These findings lead us to propose that a fully epigenetically driven mechanism might contribute to explaining the origin of certain subsets of human immunodeficiencies. Finally, we discuss the implications of these findings relevant to our understanding of the origin and evolution of PIDs, as well as the potential for future prophylactic and therapeutic interventions.

\section{Human Immunodeficiencies with Known Genetic Defects in Genes Encoding Epigenetic Modifiers}

Immune development is tightly controlled at the epigenetic level $[6,7]$. Indeed, there are many recent examples of immune or hematopoietic malfunctions in experimental knockout mice lacking known epigenetic regulators (Box 3), confirming the immunoregulatory role of these genes. However, here we focus on the molecular alterations that have already been identified in patients with PID (Figure 1), although it is likely that many of the findings in animals will be correlated with human diseases in the future.

Box 1. Basic Molecular Epigenetic Mechanisms

Epigenetics is the group of processes that control the establishment, maintenance, and inheritance of gene expression patterns without altering the germline DNA sequence [109]. They guarantee that the right genes are expressed at the right time in the right cellular context and in a heritable pattern, and, therefore, are responsible for establishing cell identity and function throughout development. At the molecular level, they are based on different chemical marks on DNA and histones (acetylation, methylation, phosphorylation, etc.), which can be added or removed by different enzymes, and can subsequently be 'read' by various effector proteins [110,111]. The combination of these marks generates a code that determines the windows and expression of genes (see Box 2).

Extreme complexity arises with the existence of active crosstalk between epigenetic marks themselves, and with transcription factors and other cellular structural proteins: changes in one epigenetic mark are usually followed by a cascade of other epigenetic alterations, often laterally propagated within chromatin.

\section{Glossary}

Choanae: posterior nasal apertures; two openings at the back of the nasal passage between the nasal cavity and throat.

Class switch recombination

(CSR): immunoglobulin class switching or isotype switching; mechanism that changes the isotype of Ig produced by a given B cell, such as from IgM to $\lg G$, or $\lg A$ or $\operatorname{lgE}$. In this process, the variable region of the Ig does not change and, therefore, the antibody retains affinity for the same antigen, but the change in the constant regions allows its interaction with different effector molecules. CSR occurs in GCs requiring AID protein function.

Common variable immunodeficiency (CVID): heterogeneous PID characterized by: (i) at least one of five criteria: increased susceptibility to infection; autoimmune manifestations; granulomatous disease; unexplained polyclonal lymphoproliferation; or affected family member with antibody deficiency; and (ii) with a marked decrease in $\lg G$, IgA, or, at least a poor antibody response to vaccines, or low switched memory B cells.

\section{Contiguous gene syndrome} (CGS): clinical phenotype caused by deletion of several consecutive genes in close proximity to one another on the chromosome. The phenotype of these patients is the result of a combination of individual defects of deleted genes, plus the potential synergic effects of their losses. Genotype/phenotype correlation: assessed relationship between a given genotype and its corresponding (usually pathological) phenotype, to identify the relationship that correlates a particular abnormality in a patient with a certain mutation.

Germinal center (GC): specific sites within the spleen and lymph nodes where mature $B$ cells proliferate, differentiate, and mutate their antibody genes in the processes of CSR and SHM.

Graft versus host disease (GvHD): arises after the reception of transplanted tissue from a genetically different individual (such as in HSCTs), where the donor's T cells in the graft recognize the recipient (the host) as a foreign antigen and attack 
Box 2. Overview of Molecular Epigenetic Regulators

Important molecular epigenetic regulators include:

- DNA methylation: chemical modification adding a methyl mark at the position 5 of cytosine in $5^{\prime} \mathrm{CpG} 3^{\prime}$ dinucleotides. The methyl group is transferred by DNA-methyltransferases (DNMTs), either de novo (DNMT3A and DNMT3B, establishing DNA methylation patterns) or via maintenance DNMTs (DNMT1, following the replication fork to copy DNA methylation patterns on the daughter strand). CpG islands are genome regions enriched in $\mathrm{CpG}$ dinucleotides, concentrating near gene promoters, where $\mathrm{CpG}$ cytosines that are unmethylated are usually associated with active gene transcription, whereas $\mathrm{CpG}$ island methylation, usually leads to gene repression. Correct $\mathrm{CpG}$ methylation patterns are essential for controlling gene expression during development and differentiation. Cytosine methylation can also be reverted mainly by proteins of the ten-eleven-translocation (TET1-3) family.

- Histone modifications: DNA is wound around histones to form chromatin; the different forms of histones can undergo many types of post-transcriptional modifications (methylation, acetylation, phosphorylation, ubiquitination, SUMOylation, etc.) affecting different residues (Lys, Ser, Thr, and Arg) in several positions along polypeptide histone chains, to different degrees (e.g., mono-, di-, and trimethylation). Most of these marks can also be removed by demethylases, deacetylases, and so on. Crosstalk between histone marks can occur within the same histone or among different ones and, therefore, the final functional outcome of histone modifications depends on the combination of all marks (forming a 'histone code').

- Chromatin remodelers: in eukaryotes, there are four families of ATP-dependent chromatin remodelers (SWI/SNF, ISWI, NURD/Mi-2/CHD, and INO80/SWR1), capable of shaping chromatin architecture by relocating nucleosomes and creating nucleosome-free regions in DNA, allowing the access of the transcription machinery.

- Noncoding RNAs are functional RNA molecules, not translated into proteins. Several structural and functional types exist, constituting a heterogeneous group, including molecules capable of regulating gene expression. They can participate, among other epigenetic functions, in chromatin remodeling and transcriptional and post-transcriptional regulation.
Immunodeficiencies due to Alterations in DNA Methylation Patterns

\section{DNMT3B in Immunodeficiency-Centromeric Instability-Facial Anomalies Syndrome 1}

ICF1 syndrome (OMIM 242860) is an autosomal recessive disorder in which patients present with immunodeficiency, facial anomalies, and instability of the pericentromeric heterochromatin of chromosomes 1, 9, and 16. The immunodeficiency is usually severe and can lead to premature death during childhood due to infections [11]. Patients normally present a decrease in serum IgG levels, and reduced numbers of $\mathrm{B}$ or $\mathrm{T}$ cells are also possible. The gene responsible for ICF1 encodes DNA de novo methyltransferase 3B (DNMT3B; Box 2 and Figure 1) [12,13]. A full knockout of Dnmt3b catalytic activity is perinatal lethal in mice [14], suggesting that mutations in patients with ICF1 are more likely to impair than to completely abolish enzymatic activity (Box 3). This is supported by the fact that mice carrying the version of Dnmt3b mutated as in humans with ICF1 are viable and show deregulated DNA methylation [15] (Box 3).

Patients with ICF1 harbor cytogenetic abnormalities affecting mainly chromosomes 1, 16, and, to a lesser extent, 9, and the hypomethylation of juxtacentromeric repeats on these chromosomes was identified before the discovery of DNMT3B as the ICF1-causing gene [16]. Patients with ICF1 present a targeted rather than global genome hypomethylation, leading to chromatin decondensation and chromosome instability $[13,17,18]$. Chromosomes 1, 6, and 9 contain the longest blocks of satellite 2 and 3 tandem repeats adjacent to their centromeres relative to other chromosomes; however, it is still unclear why the loss of the widely expressed DNMT3B selectively affects specific repetitive sequences. It might be due to the subcellular distribution or the context-specific protein interactions of DNMT3B [19], or that DNMT3B activity is more important for the methylation of sequences with a high density of CpGs than for other types of sequences, as observed for satellite 2 repeats [20]. In any case, chromatin modification studies comparing B cell lymphoblastoid cell lines (LCLs) derived from patients with ICF1 versus those from healthy controls showed that these cell lines present dysregulation of genes involved in normal lymphocyte signaling, maturation, and migration, providing a mechanistic link with the disease immunophenotype [11,21]. DNMT3B-dependent the recipient's host tissues, creating severe organ damage.

Hematopoietic stem cell transplantation (HSCT): medical procedure that involves transplanting HSCs, either from a healthy, compatible donor, or as cells from the same patient (autologous); for example, cells that have been treated to correct a particular defect. Injected cells will engraft in the bone marrow and produce new, healthy blood cells. HSCT can be used to replace diseased bone marrow that is malfunctioning and producing aberrant or insufficient blood cells.

Hyper IgM syndrome (HIGM): type of PID. People with HIGM cannot switch (see 'CSR') from the production of IgM antibodies to antibodies of the $\lg G$, IgA or $\lg E$ types: they have decreased concentrations of IgG and IgA but normal or elevated IgM in their blood. $B$ cells require active interaction with T cells to carry out CSR. HIGM can result from genetic defects interfering in the correct interaction between $T$ and $B$ cells.

Immunological memory: the capacity of the immune system to respond more rapidly and specifically when the organism is attacked again (challenged) by a previously encountered pathogen; mainly mediated by memory $\mathrm{B}$ and memory $T$ cells.

Memory B cells: see 'immunological memory'.

Somatic hypermutation (SHM): programmed process of mutation where point mutations accumulate in the variable regions of immunoglobulin genes at high rates. SHM occurs in GCs, where newly mutated lgs are positively selected to increase their affinity and specificity for antigen. SHM is initiated by the AID protein.

Transitional B cells: an intermediate differentiation stage in B cell development situated between immature $B$ cells in the bone marrow and mature $B$ cells in the spleen. 


\section{Molecular Epigenetic Defects in Human Immunodeficiencies}

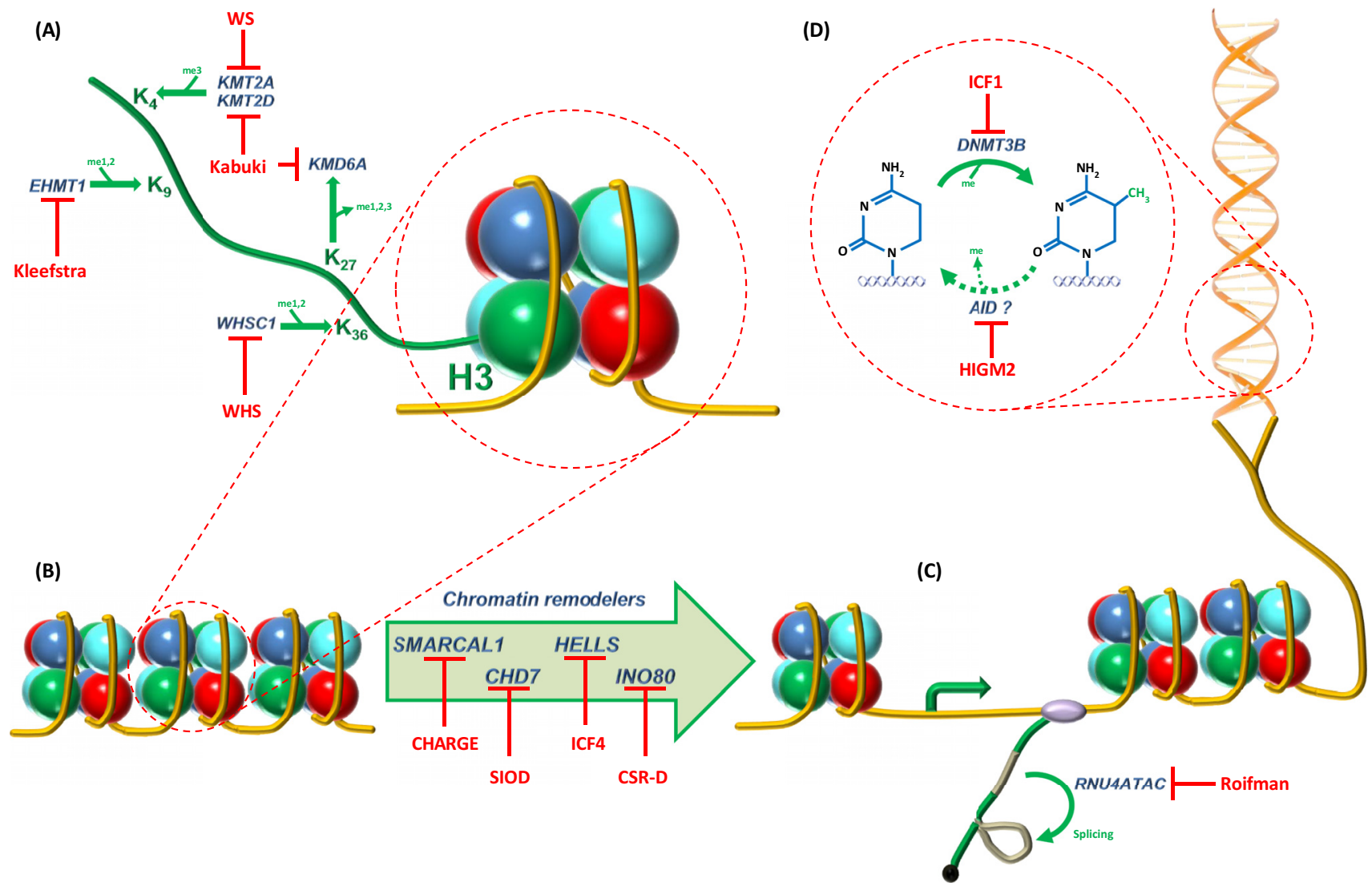

Trends in Immunology

Figure 1. General scheme of the main epigenetic regulatory mechanisms described in Boxes 1 and 2, and examples of their deregulation in human primary immunodeficiencies (PIDs). (A) Post-translational modifications in histone tails. Lysine residues (K) on histone 3 (H3) tail are shown within the context of the nucleosome, with their respective positions indicated (K4, 9, 27, or 36). The enzymes responsible for their methylation or demethylation are shown in blue, and the syndromes in which these enzymes are defective are indicated in red (WS Wiedemann-Steiner syndrome; Kabuki syndrome; WHS: Wolf-Hirschhorn syndrome; and Kleefstra syndrome). (B) Compacted nucleosomes and inaccessible chromatin. Nucleosome repositioning is required to access the DNA for transcription, and this is mediated by chromatin remodelers (see Boxes 1 and 2). The ones that have been found altered so far in human immunodeficiencies are shown in blue inside the green arrow, and the syndromes in which they are defective are indicated in red (CHARGE syndrome; ICF4: Immunodeficiency-Centromeric Instability Facial anomalies syndrome-4; SIOD: Schimke immuno-osseous dysplasia: and INO80 loss-of-function). (C) Once nucleosomes have been repositioned, DNA is accessible to the transcription machinery and RNA is synthesized. Correct splicing is essential for normal cellular function, and can be epigenetically regulated by noncoding RNAs, such as RNU4ATAC, which is inactive in Roifman syndrome, therefore leading to serious defects. (D) At the level of DNA itself, the best studied epigenetic modification is cytosine methylation, mediated by, among other enzymes (see Boxes 1 and 2) DNMT3B, which is mutated in Immunodeficiency-Centromeric Instability Facial anomalies-1 syndrome (ICF1). Cytosine methylation can also be reverted in a process initiated by several enzymes, among them activation-induced cytidine deaminase protein (AID), inactivated in Hyper-IgM syndrome 2 (HIGM2). The question mark close to AID indicates that the consequences of its ability to demethylate DNA are not yet fully understood.

methylation changes in LCLs from patients with ICF1 have been correlated with a decrease in the repressive H3K27m3 mark and an increase in the activating H3K9ac and H3K4m3 marks relative to normal LCLs, leading to increased gene expression [21]. These results show that the loss of DNMT3B function leads to hypomethylation of nonrepetitive regions of the genome 
Box 3. Animal Models of Altered Epigenetic Regulation

There are many published examples of immune malfunction and developmental alterations of the hematopoietic system in different experimental models, mainly knockout mice, in which epigenetic regulators have been eliminated or inactivated by mutation [98]. Mutations in epigenetic regulators can act as initiating oncogenic hits in many types of human solid and hematopoietic tumor, and loss-of-function mouse models tend to recapitulate parts of the human pathological phenotype, including aberrant hematopoietic differentiation [112]. Frequently, heterozygous or homozygous full loss of function of epigenetic regulators leads to serious, even lethal, developmental defects, as an indication of their essential role; this is clearly paralleled by the severity of phenotypes seen in human syndromes [113-115]. As exemplified by mouse models of ICF1 syndrome (see main text), fully knocking out epigenetic regulators in mice might point us towards the involvement of these genes in human pathologies. However, if alterations in these genes were to be found in humans, they would most likely be hypomorphic (either exonic, regulatory, or hemizygous) rather than null, since most of these genes are too important for embryonic development, and null mutations are probably incompatible with life.

and alterations in the histone code, also highlighting the interplay between DNA methylation and other epigenetic modifications.

Besides the aforementioned transcriptional changes, DNA replication is also altered in ICF1, either as a consequence of the alterations in the gene expression profile, or secondary to structural chromatin changes affecting the DNA replication machinery [22]. This might also be correlated with ICF1-intrinsic chromosomal instability, but this remains to be tested.

Approximately $40 \%$ of patients with ICF do not have mutations in DNMT3B, and constitute a group called 'ICF syndrome type 2', presenting mutations in the ZBTB24 gene, which encodes a transcriptional repressor involved in hematopoietic development [23]. ZBTB24 has a hypermethylated promoter in patients with ICF1 [24], suggesting that ZBTB24 malfunction is an underlying mechanism accounting for the similar phenotypes seen in patients with ICF1 versus those with ICF2. There is also an ICF3 subtype caused by mutation in the CDCA7 gene (involved in HSC biology by a still as yet unclear mechanism) and an ICF4 type, caused by mutation in the HELLS gene [encoding a chromatin-remodeling ATPase (Box 2) required for correct DNA methylation] $[25,26]$. Since hypomorphic DNMT3B mutations are sufficient to cause most cases of ICF1 [27], this syndrome is a paradigmatic example of how aberrant DNA methylation can be involved in immunodeficiencies.

\section{Immunodeficiencies due to Alterations in Histone Modifications}

WHSC1 in Wolf-Hirschhorn Syndrome

WHS (OMIM 194190) is caused by the loss of genetic material in the ' $p$ ' arm of chromosome 4, and is a contiguous gene syndrome [28]. Affected patients present with numerous defects, including malformations, intellectual disabilities, and convulsions. They exhibit several degrees of immunodeficiencies, including impaired antibody responses to T-independent antigens, such as lipopolysaccharides, and deficiencies in IgA and IgG2 production, and also exhibit CVID-like disease [29]. Among the genes affected by the 4p deletion, genotype/phenotype correlation studies point to the hemizygous loss of the gene encoding Wolf-Hirschhorn Syndrome Candidate-1 (WHSC1, also known as MMSET and NSD2) as the main cause for malformations and immunodeficiencies [30]. WHSC1 is a large protein with histone H3 lysine 36 (H3K36) mono- and di-methyltransferase activity, known to act as an epigenetic regulator, as shown by in vitro activity assays [31] and by in vivo studies using Whsc1-knockout mice [32] (Box 2, Figure 1). Among many other modifications, histone 3 can be mono-, di- and trimethylated in its Lysine-36. The H3K36m2 and H3K36m3 marks have been implicated in many processes associated with basic genome-wide DNA biology, including transcriptional 
elongation, DNA replication and repair, as well as establishing correct patterns of gene expression $[33,34]$.

Mouse Whsc1-knockout embryos die before birth or shortly thereafter [32]. Whsc1 was recently shown to be essential for the normal differentiation and function of lymphocytes [35]; in this study, both specification and commitment to the B cell lineage were impaired in $\mathrm{WhSC}^{-1-}$ pro-B cells, as evidenced from competitive fetal liver transplantation experiments in which normal numbers of Whsc $1^{-1-}$ progenitors were injected in a 1:1 ratio against wild-type (WT) progenitors. Relative to WT controls, Whsc $1^{-1-}$ pro-B cells also exhibited significantly reduced expression of transcription factors Ebf1 and Pax5, essential for specification and commitment to the B cell lineage at the earliest stages of B cell development [35]. At later stages, the second functional and developmental impairment of Whsc ${ }^{-1-}$ B cells was shown to occur during germinal center (GC) formation, where class switch recombination (CSR), which is necessary for the generation of new (switched) Ig isotypes, was significantly reduced relative to WT controls. This was found to occur in a Whsc1-dose-dependent manner, since CSR was more impaired in $\mathrm{Whsc}^{-/-}$than in $\mathrm{Whsc}^{+/-}$cells and, in both those cell types, was more impaired than in WT cells. This impairment was accompanied by aberrant proliferation of Whsc1-deficient B cells, as measured by BrdU-incorporation cell cycle assays, caused, in turn, by elevated replicative stress (determined by DNA fiber analysis) and by the accumulation of DNA damage (as seen by increased levels of histone $\gamma \mathrm{H} 2 \mathrm{AX}$ incorporation) [35]. These findings closely correlate with certain immunodeficiency patterns described in patients with WHS, with defective production of switched lgs suggestive of CSR impairment [29], along with a DNA replication impairment phenotype seen in immortalized cell lines derived from patient samples [36]. Impaired development of $W h s c 1^{+/-}$lymphocytes has also been reported to gradually increase with age, and this is clinically relevant because it may have important implications for the long-term prognosis of immunodeficiency in patients with WHS [35]. This Whsc1 genetically ablated mouse model provides an example of a preclinical model that can recapitulate certain aspects of the immunodeficiency seen in humans.

KMT2D and KDM6A in Kabuki Syndrome

Kabuki syndrome (OMIM 147920) is a congenital disorder affecting many organs, and is associated with a typical facial morphology, intellectual disability, skeletal abnormalities, and immune deficiencies [37]. Exome-sequencing studies revealed that, in 52-76\% of patients, the pathology is due to mutations in the KMT2D gene (encoding the histone methyltransferase Lysine-K-Methyltransferase 2D, also known as MLL2) [38] (Box 2 and Figure 1) that preferentially mediates trimethylation of histone $\mathrm{H} 3$ lysine 4 ( $\mathrm{H} 3 \mathrm{~K} 4 \mathrm{me} 3$, a gene activation mark mainly in charge of driving the transcription of constitutively expressed genes). Patients with Kabuki syndrome lacking alterations in KMT2D can present mutations (three out of 32 patients analyzed) in KDM6A [39], encoding a histone demethylase that removes mono-, di-, and trimethyl groups from H3K27me3 (a repressive mark). Therefore, the two main genes involved in Kabuki syndrome are part of the gene activation machinery, functioning to either add active epigenetic marks or remove repressive ones, and, thus, affecting many downstream genes. Nonetheless, the genetic basis of the syndrome remains unknown in $20-45 \%$ of patients [40].

Immune dysfunction is a common finding in Kabuki syndrome, since correct histone-3 methylation patterns are essential to achieving modifications in Ig genes required for B cell development and function [41]. Approximately half of the patients present with CVID-like characteristics, with decreased IgA levels, low total IgG levels, and some specific IgG subclass abnormalities [42]. Relative to healthy individuals, the numbers of memory B cells are reduced, with decreased levels of somatic hypermutation (SHM) in IgG (and, therefore, 
reduced antibody affinity) and, consequently, there is an inability to generate or maintain specific antibody responses and long-term immunological memory [42]. Several patients with Kabuki also present with autoimmune diseases, such as idiopathic thrombocytopenic purpura (ITP), hemolytic anemia, thyroiditis, or vitiligo, although the frequency of autoimmune conditions in this syndrome is unclear [43].

Finally, besides the originating mutations, and as a consequence of them, patients with Kabuki syndrome present aberrant but syndrome-specific DNA methylation abnormalities, supporting the existence of crosstalk between histone and DNA methylation systems that may be altered by inborn errors of the epigenetic machinery $[44,45]$.

\section{EHMT1 in Kleefstra Syndrome}

Kleefstra syndrome (OMIM 610253) is a neurodevelopmental disorder characterized by autism, microcephaly, and dysmorphic features. Other complications, such as congenital heart and urogenital defects and immunodeficiency, are also frequently observed [46]. Kleefstra syndrome is caused by haploinsufficiency of the gene encoding Euchromatin Histone Methyltransferase 1 (EHMT1, also known as G9a-Like Protein or GLP) [47]. Approximately $75 \%$ of patients with Kleefstra syndrome have heterozygous microdeletions at chromosome 9q34.3, whereas the remaining $25 \%$ have an intragenic EHMT1 pathogenic variant [48]. EHMT1, together with its paralog G9a, mono- and dimethylates Lys-9 on histone H3 (H3K9me1 and $\mathrm{H} 3 \mathrm{~K} 9 \mathrm{me} 2$ ) in euchromatin and, therefore, can promote heterochromatization and gene repression [49]. The effects of deregulated H3K9 methylation manifest mainly at the level of the nervous system in patients with Kleefstra syndrome, as evidenced by the mainly neurodevelopmental nature of their defects, but they can affect the whole organism, although the way in which they might interfere with immune functions remains unclear [50].

\section{KMT2A in Wiedemann-Steiner Syndrome}

Wiedemann-Steiner syndrome (OMIM 605130) is an autosomal dominant syndrome in which patients present with a wide spectrum of alterations, including intellectual disability, developmental delay, and immunodeficiency. It is caused in almost $100 \%$ of the identified cases by heterozygous mutations in the KMT2A gene (encoding Lysine Methyltransferase $2 \mathrm{~A}$, also called mixed-lineage leukemia, $M L L$ ) [51], encoding a H3K4 methyltransferase involved in hematopoiesis by governing the expression of key regulators, such as the HOX and WNT genes [52]. Several types of immune alterations have been described in patients with Wiedemann-Steiner syndrome, including immunodeficiency accompanied by low concentrations of Igs, and frequent urinary tract, respiratory, and ear infections [53]. In fact, PID resembling early-onset CVID might be the first indication of Wiedemann-Steiner syndrome, despite nonimmunological characteristics not yet being apparent [54].

Thus, WHS, Kabuki, Kleefstra, and Wiedemann-Steiner syndromes have been linked to altered histone methylation patterns that can lead to defective antibody and lg production in humans.

\section{Immunodeficiencies due to Alterations in Chromatin Remodelers}

SMARCAL1 in Schimke Immuno-Osseous Dysplasia

SIOD (OMIM 242900) is an autosomal recessive multisystem disorder characterized by bone dysplasia, growth deficiency, defective cellular immunity, and progressive renal failure [55]. Patients are predisposed to viral and bacterial infections and present with decreased numbers of $\mathrm{CD}^{+} \mathrm{T}$ cells in peripheral blood relative to healthy individuals [56]. Ex vivo, $\mathrm{T}$ cell proliferation is impaired, and Ig levels are abnormal in two-thirds of patients, although B cell numbers are normal [56]. 
SIOD is caused by mutations in SMARCAL1 (encoding SWI/SNF-Related, Matrix Associated, Actin-Dependent Regulator of Chromatin, Subfamily A Like 1) [57] (Figure 1), which encodes an ATP-dependent DNA annealing helicase that maintains genomic integrity by participating in DNA replication fork restart and DNA repair [58]. The IL7Ra promoter was found to be hypermethylated in T cells from peripheral blood of patients with SIOD, therefore diminishing IL-7 receptor alpha chain (IL7R $\alpha$ ) expression and rendering T cells less responsive to IL-7, a stimulation pathway that is necessary for their development [59]. In human SIOD skin fibroblasts, as well as in Drosophila or mice defective in SMARCAL 1 orthologs, there is an alteration in the expression of many genes (versus control WT cells); however, in both mice and Drosophila, this alteration is insufficient to cause disease in the absence of additional environmental or genetic insults [60]. This has led to the suggestion that the incomplete penetrance of SIOD in humans is due to the fact that SMARCAL1 deficiency may need to be complemented by other genetic or environmental factors in order to alter gene expression, and that the function of SMARCAL1 annealing helicase activity is to maintain DNA topology in order to buffer variability in gene expression and, as a result, diminish the penetrance of pathologic traits arising from environmental and genetic insults [60].

\section{INO80 in Class Switch Recombination Defects}

Class-switch recombination defects (CSR-D) are rare PIDs characterized by impaired generation of switched immunoglobulin isotypes. CSR-Ds might be caused by impaired cooperation between $B$ and $T$ cells or by several types of intrinsic $B$ cell defects, which have not always been identified [61]. Exome sequencing of two patients with CSR-D identified recessive, nonsynonymous coding variations in the INO80 gene [61]. INO80 is the catalytic ATPase subunit of the INO80 chromatin-remodeling complex (Box 2 and Figure 1) and participates in many processes affecting DNA biology, including transcriptional regulation, DNA repair, and DNA replication [62]. Enforced re-expression of WT INO80 in patient fibroblasts was found to correct cellular hypersensitivity to $\gamma$-irradiation [61]. Furthermore, in mouse B-lymphoid $\mathrm{CH} 12-\mathrm{F} 3$ cells, the INO80 complex was shown to accumulate at switch regions of Ig loci and, in addition, INO80 downregulation via small hairpin (sh)RNA in the same cells led to impaired CSR, indicating that INO80-mediated chromatin-remodeling activity was required for correct resolution of the CSR process, probably at the level of DNA repair, although the potential mechanism remains to be tested [62].

\section{CHD7 in CHARGE Syndrome}

CHARGE syndrome (OMIN 214800) is an autosomal dominant disorder characterized by the conjunction of multiple birth defects (coloboma of the eye, heart defects, atresia of the choanae, retardation of growth, genital and ear abnormalities). It presents a phenotypic overlap with Kabuki and other immunodeficiency syndromes [63], and patients also exhibit global changes in DNA methylation profiles [44]. However, CHARGE syndrome is caused by loss-of-function mutations in the CHD7 gene, encoding a chromodomain helicase DNA-binding protein, which is an ATP-dependent chromatin remodeler (refer to Box 2 and Figure 1) [64]. CHD7 is the only gene currently known to be associated with CHARGE syndrome, and pathogenic variants in this gene are found in $\sim 65-70 \%$ of patients [65]. Patients with CHARGE can present with immunodeficiency, mainly associated with low peripheral $\mathrm{T}$ cell numbers $\left(\mathrm{CD}^{+}, \mathrm{CD}^{+}\right.$, and/or $\mathrm{CD} 8^{+} \mathrm{T}$ cells) relative to healthy individuals [66]; however, this is likely not due to a $\mathrm{T}$ cell-autonomous defect, since thymic aplasia with severely reduced thymic function can be present in patients with CHARGE; therefore, the main role of $\mathrm{CHD} 7$ malfunction in $\mathrm{T}$ cells remains to be elucidated. 
Hence, together with SIOD and INO80 loss-of-function, CHARGE syndrome constitutes an example of how alterations in proteins controlling chromatin remodeling can impact the effectiveness of the immune system in affected patients.

\section{Immunodeficiencies due to Alterations in Noncoding RNAs}

Due to the heterogeneous nature and functions of noncoding (nc)RNAs (Boxes 1 and 2), the recent identification of many of these, and the fact that a variety of important ncRNA subtypes do not have bona fide epigenetic function, there are not many examples of these that have been implicated in contributing to aberrant immune function in PIDs. However, Roifman syndrome (OMIM 616651) falls into this class and provides a proof-of-principle of the possibility that ncRNAs harbor an epigenetic function that can impact human immunodeficiency. Roifman syndrome is an autosomal recessive disorder characterized by skeletal dysplasia, immunodeficiency, retinal dystrophy, and developmental delay [67]. In all patients studied so far, the syndrome is caused by compound heterozygous mutations in the small nuclear RNA gene RNU4ATAC, necessary for U12-type intron splicing [68]. This mutation disrupts correct intron splicing and leads to myriad alterations, including immunodeficiency [54]. By analyzing the immune and hematopoietic systems of three patients with Roifman syndrome from two unrelated kindreds with RNU4ATAC variants [69], specific splicing defects in key genes (see later) were identified that might contribute to explaining the immune phenotypes seen in patients. Indeed, patients with Roifman syndrome present a severe reduction in peripheral, circulating $B$ cell numbers, with a $B$ cell differentiation block at the transitional B cell stage compared with healthy individuals [69]. RNA-sequencing studies identified a splicing defect in the gene encoding mitogen-activated protein kinase 1 (MAPK1) [69]. Since MAPK1-MAPK3 heterodimers are required for survival signaling necessary for B cell differentiation [70], this intrinsic defect might contribute to the observed phenotype, although it does not exclude the presence of other B cell-intrinsic or extrinsic defects in differentiation and survival caused by loss of function of RNU4ATAC and its associated minor splicing complex [69].

\section{Proteins with a Secondary Epigenetic Function Linked to Primary Immunodeficiencies}

Recent research focusing on altered proteins in immunodeficiencies has identified new molecular functions for these proteins. These findings might provide new insights into immune dysfunction in certain PIDs, the molecular mechanisms of which were originally thought to be independent of any epigenetic modifications.

\section{WASP in Wiskott-Aldrich Syndrome}

One clear example is Wiskott-Aldrich syndrome (WAS, OMIM 301000), an X-linked disorder characterized by microthrombocytopenia, eczema, and recurrent infections [71]. WAS is caused by mutations affecting the WAS protein (WASP), the main function of which is to relay signals from the surface of the cell to the actin cytoskeleton. WASP deficiency results in significant abnormalities of T-lymphocyte numbers and function [72]; T cells from patients with WAS present an absent or impaired function of WASP, along with impaired T cell differentiation and a decreased ability to be activated and to respond to the environment, relative to controls [71]. However, besides its cytoplasmic function, WASP also has nuclear activity and is capable of modulating the expression of the TBX21 gene [73]. TBX21 (or T-Bet: T-box protein expressed in $\mathrm{T}$ cells) was originally described as a transcription factor essential for the commitment of $\mathrm{T}$ helper (Th) cells to the Th1 lineage, by directly activating the transcription of the gene encoding IFN- $\gamma$ [74]. At the same time, TBX21 modulates Th2 cytokines to result in decreased Th2 development [74]. Later studies reported that TBX21 has multiple roles in many 
other immune cell types, including B cells, dendritic cells, and natural killer (NK) cells, and, therefore, is deemed essential for the development and coordination of both innate and adaptive immune responses [75]. In WAS, WASP modulates the expression of TBX21 by associating with $\mathrm{H} 3 \mathrm{~K} 4$ methyltransferase RBBP5 and $\mathrm{H} 3 \mathrm{~K} 9 / \mathrm{H} 3 \mathrm{~K} 36$ demethylase JMJD2A, the enzymatic activities of which are required for activating chromatin at the TBX21 proximal promoter [73], supporting the existence of a nuclear mechanism underlying deregulated $T$ cell immunity in human WAS.

AID in immunodeficiency syndrome with hyper-lgM, type 2

Another example of a protein with an epigenetic role 'additional' to its main function is that of activation-induced cytidine deaminase protein (AID). AID can catalyze cytosine-to-uracil deaminations on single-stranded DNA mainly at genes encoding Igs during the GC reaction, creating $U: G$ mismatches that, when repaired by different means, can lead to CSR and SHM $[76,77]$. Accordingly, inactivating AID mutations cause immunodeficiency syndrome with hyper-IgM, type 2 (HIGM2) (OMIM 605258) [78]; patients with HIGM2 are susceptible to bacterial infections because their B cells cannot perform CSR and SHM [78]. However, AID, harboring deaminase activity, when acting on methylated cytosines, can also lead to effective DNA demethylation [79] and, therefore, might have a role in the demethylation of specific loci and the activation of specific genes relevant in immunodeficiencies (reviewed in [80]). It is clear that the phenotype of patients with HIGM2 is primarily due to an impaired production of switched and hypermutated antibodies; nonetheless, mouse Aid can regulate DNA methylation in HSCs as well as during myeloid/erythroid differentiation, and Aid loss in knockout mice leads to myeloid cell expansion and reduction in the numbers of erythroid progenitors, with dysregulated expression of Cebpa and Gata1, encoding key myeloid/ erythroid lineage-specific transcription factors [81]. Similarly, shRNA-mediated knockdown of AID in human bone marrow cells skews their differentiation toward the myelomonocytic lineage [81]. During reprogramming of primary mouse embryonic fibroblast (MEF) to induced pluripotent stem cells, bisulfite genome-wide analysis of DNA demethylation showed that reprogramming appears to require Aid-mediated downregulation of Uhrf1 protein, an essential component of the DNA methylation maintenance machinery, although this appears to be independent on Aid deaminase activity [82]. In addition, AID has been shown to be a driver of epigenetic heterogeneity in B cell lymphomas, since ectopic Aid overexpression causes more aggressive disease in Bcl2-driven murine lymphomas, and this phenotype is not associated with increased Aid-mediated somatic mutation, but with elevated cytosine methylation heterogeneity [83]. Therefore, through some of the aforementioned mechanisms and/or others still unknown, a potential (albeit accessory) AIDmediated epigenetic component in patients with HIGM2 may be significant and cannot be completely discarded.

\section{Epigenetic Alterations without a Known Genetic Origin in Human Immunodeficiency}

Epigenetic modifications can be changed (reprogrammed) in response to either internal or environmental stimuli in the absence of genetic mutations. This can happen, as in the case of lymphocyte development, in the context of normal cellular differentiation and, even more, it is in fact necessary for the definition of cellular identity [6]. However, epigenetic reprogramming can also be triggered by environmental exposures [10], and the altered epigenetic patterns can be self-perpetuating. From studies in cancer biology, we have learned that epigenetic alterations have an essential role in the origin and evolution of most types of tumor; for many years, only bona fide genetic mutations affecting the DNA sequence were considered important as molecular culprits behind cancer origin or progression $[8,84]$. However, we now know that 
(A)

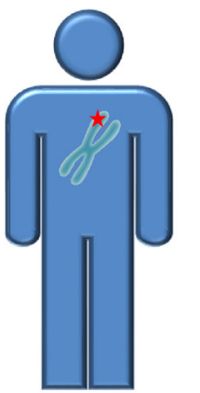

Congenital genetic mutation

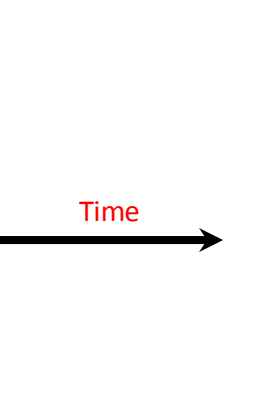

Epigenetic priming

(B)

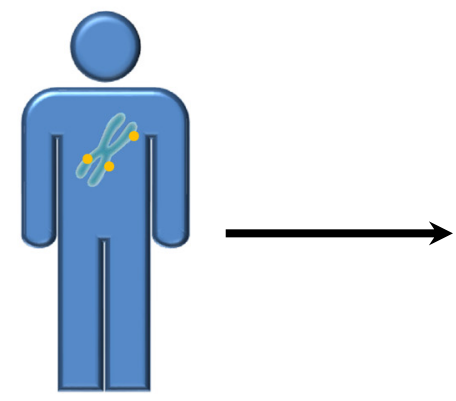

Genetic predisposition

(c)

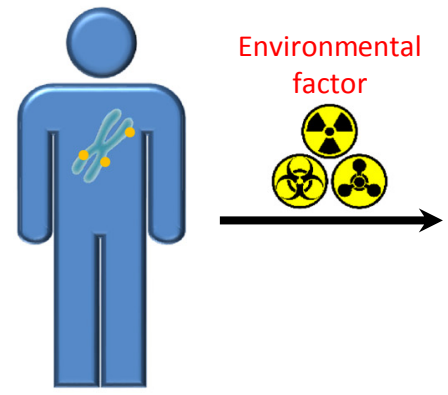

Genetic predisposition

(D)

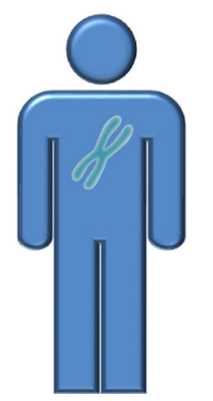

Environmental

Healthy

Immunodeficiency
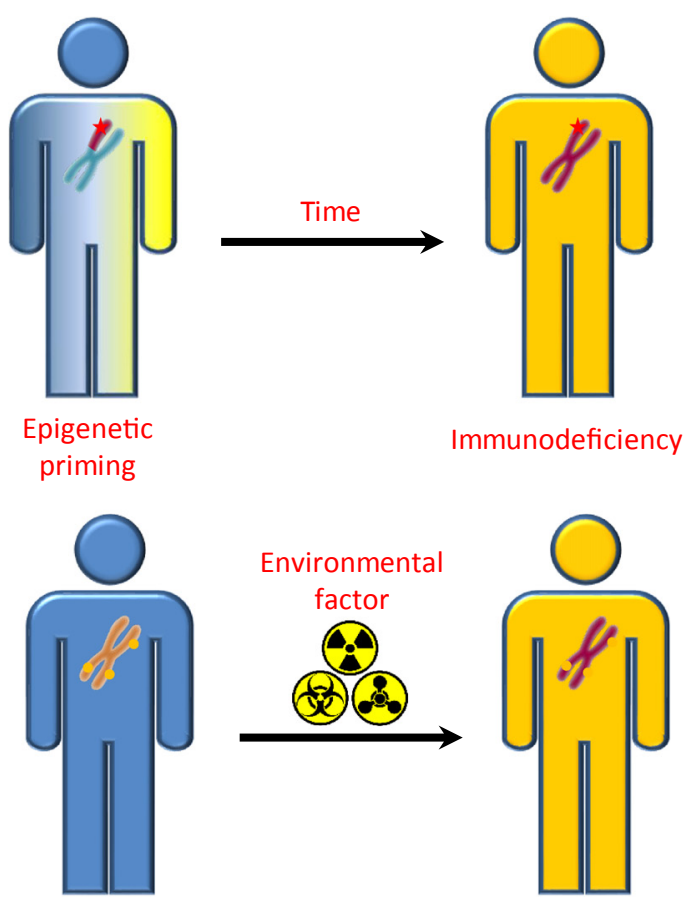

Epigenetic priming

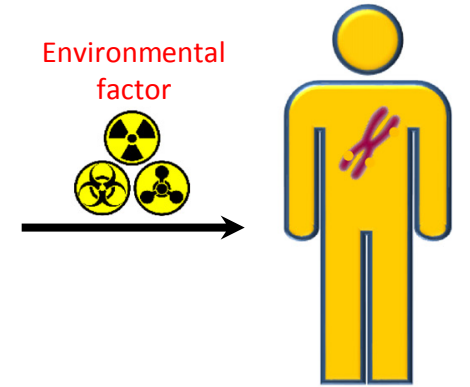

Immunodeficiency

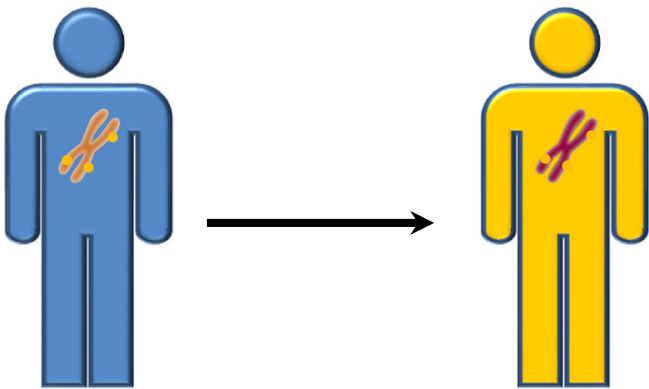

Epigenetic priming

Immunodeficiency

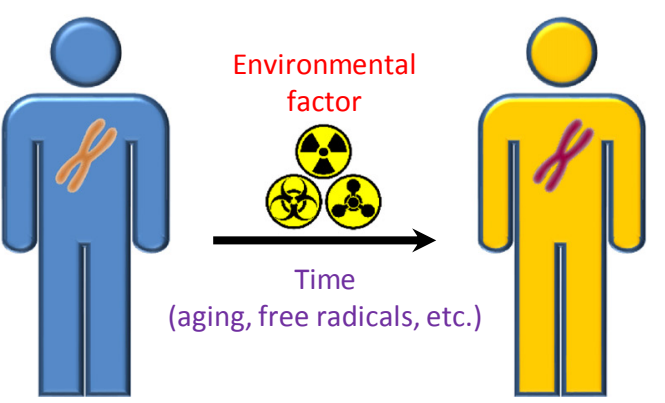

Epigenetic priming

Immunodeficiency

Trends in Immunology

Figure 2. Potential Roles for Epigenetic Priming in Human Immunodeficiencies. Although the underlying (Figure legend continued on the bottom of the next page.) 
epigenetic reprogramming is an essential part of tumor pathogenesis and maintenance $[9,85]$. Furthermore, it is known that even the earliest stages of tumor initiation can almost fully be epigenetically mediated [86-89].

The findings that we have described above open the possibility of delineating a parallel picture for our understanding of a subset of human PIDs (Figure 2), where epigenetic alterations are involved in the development and/or evolution of PIDs. We have seen that different types of alteration in essential epigenetic regulators can cause severe malfunctions of the immune system, although the specific mechanisms can vary among diseases, and many remain unknown. In addition, we have provided examples of how proteins with well-established activities can also have epigenetic functions that might be important in immunodeficiencies. The application of next-generation sequencing (NGS) techniques is allowing the diagnosis of new cases and the identification of new genes involved in immunodeficiencies [90]. Nevertheless, there are many cases where causative mutations cannot be identified $[90,91]$. In fact, for the most frequent type of PIDs, CVID, NGS approaches have only identified potentially disease-causing mutations in $30-65 \%$ of patients [92,93]. These percentages have led to the conclusion that a significant proportion of CVIDs might be due to polygenic causes, in which the combination of many genetic variants can lead to the appearance of different immunodeficiencies of variable characteristics [91,94-96].

By analogy with cancer, a step further takes us to the additional, nonexcluding, possibility of the existence of epigenetic anomalies that condition or prime the development of PIDs, and do not have their origin in genetic mutations of epigenetic regulatory genes, but are in fact triggered by internal or environmental reprogramming events (Figure 2). Along these lines, in a recent study, high-throughput DNA methylation analyses in monozygotic twins discordant for CVID revealed a gain of DNA methylation in affected B cells compared with those from a healthy sibling [97]. A confirmatory study in a cohort of sporadic CVID cases revealed that critical B cell genes, such as PIK3CD, BCL2L1, RPS6KB2, TCF3, or KCNN4, showed an impaired capacity for demethylating and upregulating their transcriptional expression in B cell transition from naive to memory cells in CIVD [97]. These results, together with data from knockout mice (Box 3 [98]) and other reports discussed in this review, lead us to propose that a fully epigenetic mechanism might contribute to explaining the origin of certain subsets of human immunodeficiencies (Figure 2).

epigenetic mechanism initially altered might vary (DNA methylation, histone modifications, or chromatin remodeling), its involvement in the immunodeficiency pathology can follow a series of similar steps. (A) A defined genetic mutation inactivates a given epigenetic regulator (or a protein with an indirect epigenetic effect). This leads to epigenetic reprogramming that modifies the pattern of gene expression of the affected cells and, therefore, their development and/or function. If the penetrance of the phenotype associated with this mutation and/or syndrome is high, then the appearance of the immunodeficiency is just a matter of time (variable depending on the disease). If the penetrance is lower, then the appearance of the immunodeficiency may be conditioned by other modifying genes or by environmental exposures (infections or other agents, see later). (B) In the absence of a defined genetic mutation, the combination of specific gene variants might prime the epigenomes of certain individuals and make them more susceptible to external agents that might trigger immunodeficiency. This dependence on external factors could explain the differences found in identical twins. (C) Conversely, this combination of specific gene variants might make the individual more susceptible to changes caused by external agents, and these changes can in turn lead to an epigenetic priming that ultimately causes immunodeficiency. (D) Finally, in a 'completely healthy' individual, exposure to environmental factors in early life (pathogens, radiation, chemicals, etc.) might trigger epigenetic changes that render this individual more susceptible to exposure to external or endogenous agents later in life, which will trigger immunodeficiency. 


\section{Translational Implications of Epigenetic Reprogramming in PIDs}

The existence of epigenetic deregulation as a proposed mechanism of pathogenesis in certain PIDs might have diagnostic, prognostic, prophylactic, and even therapeutic implications for clinical management (Figure 3). On the one hand, epigenetic modifications can be triggered by exposure to many different environmental agents (chemicals, radiation, infections, our own microbiome, etc.) and also by endogenous causes, such as exercise or aging [99]. The immune

(A)

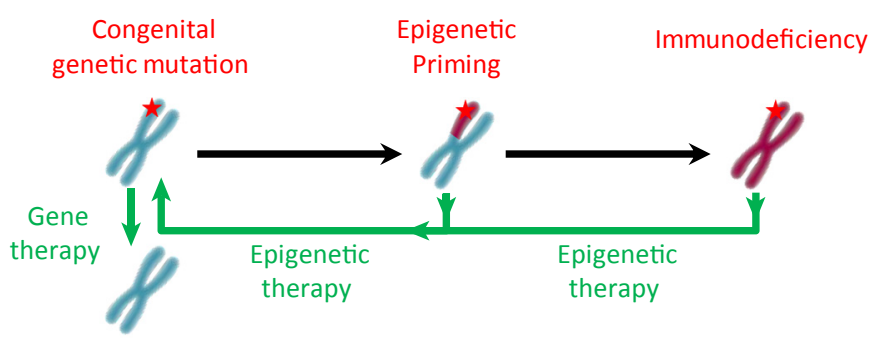

(B)

$\begin{array}{ccc}\text { Genetic } & \text { Epigenetic } & \text { Immunodeficiency } \\ \text { predisposition } & \text { priming }\end{array}$

Diagnosis

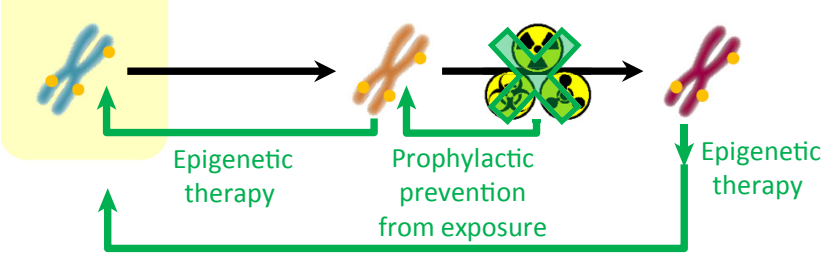

(C)

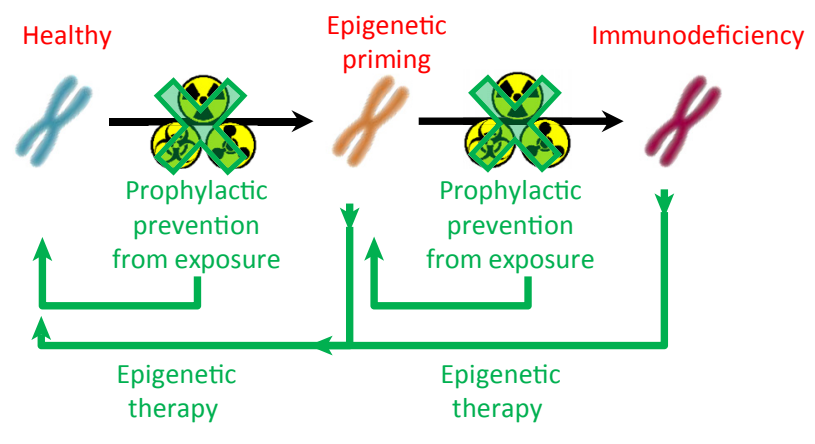

Trends in Immunology

Figure 3. Prophylactic and Therapeutic Implications of Epigenetic Modulation in Immunodeficiencies. (A) An inactivating mutation could be corrected by using some of the diverse existing gene therapy approaches. The alternative would be to revert the pathological epigenetic priming and restore normal physiological cellular development and function. Proof-of-principle experiments in animal models will have to demonstrate whether aberrant epigenetic reprogramming can be reverted to normal conditions in vivo, something that likely varies among diseases. Afterwards, either highly specific epigenetic drugs or targeted gene therapy would be required to guide therapeutic reprogramming. (B) In the context of susceptibility, the first key aspect would be the diagnosis and identification of such predisposition, something that is still in its infancy in most cases. Once an individual has been found to be susceptible to a certain disease or to a certain exposure, then prophylactic measures might be undertaken to avoid such exposure. Alternatively or complementarily, epigenetic therapies would need to be established to potentially prevent or to revert pathological reprogramming. (C) Finally, if specific exposures at defined developmental points are shown to promote immunodeficiency in healthy, nonsusceptible individuals, then preventive measures might be implemented to avoid such consequences. Again, alternatively or complementarily, epigenetic therapies might be established to putatively prevent or to revert pathological reprogramming. 
system, by its very nature, is especially sensitive to environmental exposures (which, in instances such as chemotherapy, radiation, HIV infection, or pesticides, can be associated with, or causal to, secondary immunodeficiencies that are not genetic in origin), triggering abnormalities, such as leukemia and autoimmunity, the etiology of which has been linked to clear epigenetic regulation [9,100-102]. A deeper knowledge of the precise epigenetic mechanisms by which such exposures might contribute to the appearance of certain PIDs might allow the future exploration of approaches aimed at recovering potential developmental or functional immune cell impairment (Figure 3). Numerous questions remain (see Outstanding Questions), and extensive, robust testing will be required to address these.

The potentially polygenic origin of still unidentified PIDs might render some patients more susceptible to epigenetic reprogramming of their immune system as a response to certain stimuli (e.g., infections, medication, or malnutrition). Moreover, the identification of variants or SNPs associated with such susceptibilities might allow a putative prognostic evaluation, or clinical and analytical follow-up, in individuals at risk for developing a PID disease with full penetrance (see Outstanding Questions).

\section{Concluding Remarks}

The only curative procedure for severe and life-threatening PIDs so far is the correction of the gene defect in HSCs, either by transplantation of allogenic donor-derived cells or the correction of autologous stem cells by gene therapy. Predominantly, antibody deficiencies affecting the B cell differentiation and maturation without a severe combined $T$ cell defect are not suitable for HSCT due to severe complications still inherent to the procedure, such as infections and graft versus host disease (GvHD) [103,104]. In addition, gene therapy developments are understandably mostly focused on severe PIDs. When a genetic defect interferes to some degree with embryonic development (especially in syndromic immunodeficiencies), alterations in morphogenesis and organogenesis, even if compatible with life, are in most cases irreversible with current technology. However, some of the functional defects specific to a given cellular type, or those affecting systems in which morphogenesis is not so important for function (e.g., the immune system), might be amenable to correction using appropriate strategies in the right time window $[105,106]$. Evidently, these tests will have to be first performed as proof-ofprinciple experiments in animal models, and their translation to the clinic will be slow and difficult (see Outstanding Questions). However, it will be important to ascertain the degree of reversibility (if any) to a normal developmental epigenome along the different stages of cellular differentiation in animal models and in certain immunodeficient patients. Indeed, for some subgroups of PIDs, epigenetic modifiers with a potential role in reversing the cellular defects could constitute the first 'curative' possibility. Therefore, we need to better understand the molecular underpinnings of the persistence of an aberrant epigenetic pattern in immunodeficiencies to explore the possible potential of reversion to a normal, or less dysfunctional, profile. In experimental systems, certain cancer cells can be reprogrammed back to nontumoral fates, losing their malignant status via administration of epigenetic drugs, or by means of gene reprogramming [9]. Therefore, it is conceivable that either epigenetic drugs or gene therapy approaches targeted in the right cellular compartments and during the right developmental stage might lead to the reversion of the immunodeficient epigenetic program to a normal, functional one. Potential future epigenetic drugs might be similar to existing drugs, acting in a more or less genome-wide manner over a given epigenetic mark, or might be newly developed targeted epigenetic modifiers capable of altering epigenetic marks, not in a global way, but rather, in a more precise location-specific manner; this might be achieved by the use of different (epi)genome-editing tools, including zinc finger proteins, transcription activator-like effectors (TALES), or the CRISPR-Cas9 system [107].

\section{Outstanding Questions}

To what extent are PIDs of genetic origin only? Can environmental (exogenous or endogenous) exposure be a trigger of an underlying hidden (epi) genetic condition? If so, what is the contribution of each exposure type to the development of disease?

Can we identify hypomorphic but nonlethal mutations in (epi)genetic regulators that are responsible for immunodeficiencies? Can we distinguish them from normal variants? Can they act alone, or do they require collaborating variants and/or mutations?

In the absence of obvious germline or somatic mutations, can we identify genetic associations of polymorphisms indicating predisposition to specific PIDs? Can we identify the main genetic contributors to specific PIDs? Can we stratify the population according to polygenic risk scores? Will it be possible to implement effective prophylactic measures to prevent or delay the appearance of symptoms in susceptible individuals?

How can we generate faithful animal models reflecting all the complexity of human PIDs? Can proof-of-principle experiments performed in such models be translated to humans? Will these models be good proxies to study the role of environmental exposure in the development of PIDs?

Can epigenetic alterations associated with PIDs be reverted once they have already been established? Could this be done pharmacologically, or are more complex gene therapy approaches required? What will the developmental and cellular windows for therapeutic intervention be? 
Finally, there are other pathologies of immune cells with a strong epigenetic component, including, for example, leukemias, certain autoimmune disorders, or viral infections (where viruses often hijack the epigenetic mechanisms of the immune cell to subvert immune function) [108]. Therefore, we anticipate that therapeutic advances obtained studying the epigenetics of immunodeficiencies might be applicable to other pathologies, and vice versa. It will be remarkable to follow these advances in the years to come.

\section{Acknowledgments}

We are indebted to all members of our group, to María Gómez-Vicentefranqueira and to Isidro Sanchez-García for useful discussions and for critical reading of the manuscript, and to Vasco Barreto for useful discussions. Research at C.C.'s laboratory was partially supported by FEDER, the 'Fondo de Investigaciones Sanitarias/Instituto de Salud Carlos III' (PI14/ 00025), MINECO (SAF2017-83061-R), the 'Fundación Ramón Areces', the 'Federación Española de Enfermedades Raras', the 'Asociación Muévete por los que no Pueden', and a Research Contract with the 'Fundación Síndrome de WolfHirschhorn o 4p-'. Institutional grants from the 'Fundación Ramón Areces' and 'Banco de Santander' to the CBMSO are also acknowledged. Research at E.L.G. laboratory was partially supported by FIS PI16/01605 (Fondo de Investigación Sanitaria Instituto de Salud Carlos III, Madrid, Spain; FEDER), Lucía del Pino Molina received an EFIS-IL Short Term Fellowship for a short stay in Erasmus MC, Rotterdam (NL).

\section{References}

1. Picard, C. et al. (2018) International Union of Immunological Societies: 2017 Primary Immunodeficiency Diseases Committee Report on Inborn Errors of Immunity. J. Clin. Immunol. 38, 96-128

2. Bousfiha, A. et al. (2018) The 2017 IUIS phenotypic classification for primary immunodeficiencies. J. Clin. Immunol. 38, 129-143

3. Liadaki, K. et al. (2013) New facets of antibody deficiencies. Curr. Opin. Immunol. 25, 629-638

4. Jones, G.L. et al. (2018) What is the burden of immunoglobulin replacement therapy in adult patients with primary immunodeficiencies? A systematic review. Front. Immunol. 9, 1308

5. Marciano, B.E. and Holland, S.M. (2017) Primary immunodeficiency diseases: current and emerging therapeutics. Front. Immunol. 8, 937

6. Busslinger, M. and Tarakhovsky, A. (2014) Epigenetic control of immunity. Cold Spring Harb. Perspect. Biol. 6, a019307

7. Martin-Subero, J.I. and Oakes, C.C. (2017) Charting the dynamic epigenome during B-cell development. Semin. Cancer Biol. 51, 139-148

8. Ntziachristos, P. et al. (2016) Emerging concepts of epigenetic dysregulation in hematological malignancies. Nat. Immunol. 17 , 1016-1024

9. Vicente-Duenas, C. et al. (2018) Epigenetic priming in cancer initiation. Trends Cancer 4, 408-417

10. Tzika, E. et al. (2018) Epigenetics and metabolism in health and disease. Front. Genet. 9, 361

11. Ehrlich, M. et al. (2001) DNA methyltransferase 3B mutations linked to the ICF syndrome cause dysregulation of lymphogenesis genes. Hum. Mol. Genet. 10, 2917-2931

12. Hansen, R.S. et al. (1999) The DNMT3B DNA methyltransferase gene is mutated in the ICF immunodeficiency syndrome. Proc. Natl. Acad. Sci. U. S. A. 96, 14412-14417

13. Xu, G.L. et al. (1999) Chromosome instability and immunodeficiency syndrome caused by mutations in a DNA methyltransferase gene. Nature 402, 187-191

14. Okano, M. et al. (1999) DNA methyltransferases Dnmt3a and Dnmt3b are essential for de novo methylation and mammalian development. Cell 99, 247-257

15. Ueda, Y. et al. (2006) Roles for Dnmt3b in mammalian development: a mouse model for the ICF syndrome. Development 133, $1183-1192$

16. Jeanpierre, M. et al. (1993) An embryonic-like methylation pattern of classical satellite DNA is observed in ICF syndrome. Hum. Mol. Genet. 2, 731-735
17. Brun, M.E. et al. (2011) Heterochromatic genes undergo epige netic changes and escape silencing in immunodeficiency, centromeric instability, facial anomalies (ICF) syndrome. PLoS One 6, e19464

18. Yehezkel, S. et al. (2008) Hypomethylation of subtelomeric regions in ICF syndrome is associated with abnormally short telomeres and enhanced transcription from telomeric regions. Hum. Mol. Genet. 17, 2776-2789

19. Bachman, K.E. et al. (2001) Dnmt3a and Dnmt3b are transcriptional repressors that exhibit unique localization properties to heterochromatin. J. Biol. Chem. 276, 32282-32287

20. Gowher, H. and Jeltsch, A. (2002) Molecular enzymology of the catalytic domains of the Dnmt3a and Dnmt3b DNA methyltransferases. J. Biol. Chem. 277, 20409-20414

21. Jin, B. et al. (2008) DNA methyltransferase 3B (DNMT3B) mutations in ICF syndrome lead to altered epigenetic modifications and aberrant expression of genes regulating development, neurogenesis and immune function. Hum. Mol. Genet. 17, 690-709

22. Lana, E. et al. (2012) DNA replication is altered in immunodeficiency centromeric instability facial anomalies (ICF) cells carrying DNMT3B mutations. Eur. J. Hum. Genet. 20, 1044-1050

23. de Greef, J.C. et al. (2011) Mutations in ZBTB24 are associated with immunodeficiency, centromeric instability, and facial anom alies syndrome type 2. Am. J. Hum. Genet. 88, 796-804

24. Heyn, H. et al. (2012) Whole-genome bisulfite DNA sequencing of a DNMT3B mutant patient. Epigenetics 7, 542-550

25. Thijssen, P.E. et al. (2015) Mutations in CDCA7 and HELLS cause immunodeficiency-centromeric instability-facial anomalies syndrome. Nat. Commun. 6, 7870

26. Velasco, G. et al. (2018) Comparative methylome analysis of ICF patients identifies heterochromatin loci that require ZBTB24, CDCA7 and HELLS for their methylated state. Hum. Mol. Genet. $27,2409-2424$

27. Gatto, S. et al. (2017) ICF-specific DNMT3B dysfunction interferes with intragenic regulation of mRNA transcription and alternative splicing. Nucleic Acids Res. 45, 5739-5756

28. Battaglia, A. et al. (2015) Wolf-Hirschhorn syndrome: a review and update. Am. J. Med. Genet. C Semin. Med. Genet. 169 216-223

29. Hanley-Lopez, J. et al. (1998) Antibody deficiency in WolfHirschhorn syndrome. J. Pediatr. 133, 141-143

30. Derar, N. et al. (2018) De novo truncating variants in WHSC recapitulate the Wolf-Hirschhorn (4p16.3 microdeletion) 
syndrome phenotype. Genet. Med. Published online June 11, 2018. http://dx.doi.org/10.1038/s41436-018-0014-8

31. Kuo, A.J. et al. (2011) NSD2 links dimethylation of histone H3 at lysine 36 to oncogenic programming. Mol. Cell 44, 609-620

32. Nimura, K. et al. (2009) A histone H3 lysine 36 trimethyltransferase links Nkx2-5 to Wolf-Hirschhorn syndrome. Nature 460, 287-291

33. Wagner, E.J. and Carpenter, P.B. (2012) Understanding the language of Lys36 methylation at histone H3. Nat. Rev. Mol. Cell Biol. 13, 115-126

34. Streubel, G. et al. (2018) The H3K36me2 methyltransferase Nsd1 demarcates PRC2-mediated H3K27me2 and H3K27me3 domains in embryonic stem cells. Mol. Cell 70, 371-379. e375

35. Campos-Sanchez, E. et al. (2017) Wolf-Hirschhorn syndrome candidate 1 is necessary for correct hematopoietic and B cell development. Cell Rep. 19, 1586-1601

36. Kerzendorfer, C. et al. (2013) Meier-Gorlin syndrome and WolfHirschhorn syndrome: two developmental disorders highlighting the importance of efficient DNA replication for normal development and neurogenesis. DNA Repair (Amst) 12, 637-644

37. Cheon, C.K. and Ko, J.M. (2015) Kabuki syndrome: clinical and molecular characteristics. Korean J. Pediatr. 58, 317-324

38. Ng, S.B. et al. (2010) Exome sequencing identifies MLL2 mutations as a cause of Kabuki syndrome. Nat. Genet. 42, 790-793

39. Lederer, D. et al. (2012) Deletion of KDM6A, a histone demethylase interacting with MLL2, in three patients with Kabuki syndrome. Am. J. Hum. Genet. 90, 119-124

40. Banka, S. et al. (2012) How genetically heterogeneous is Kabuki syndrome?: MLL2 testing in 116 patients, review and analyses of mutation and phenotypic spectrum. Eur. J. Hum. Genet. 20, 381-388

41. Daniel, J.A. and Nussenzweig, A. (2012) Roles for histone H3K4 methyltransferase activities during immunoglobulin class-switch recombination. Biochim. Biophys. Acta 1819, 733-738

42. Lindsley, A.W. et al. (2016) Defects of B-cell terminal differentiation in patients with type-1 Kabuki syndrome. J. Allergy Clin. Immunol. 137, 179-187

43. Ming, J.E. et al. (2005) Autoimmune disorders in Kabuki syndrome. Am. J. Med. Genet. A 132A, 260-262

44. Butcher, D.T. et al. (2017) CHARGE and Kabuki syndromes: gene-specific DNA methylation signatures identify epigenetic mechanisms linking these clinically overlapping conditions. Am. J. Hum. Genet. 100, 773-788

45. Sobreira, N. et al. (2017) Patients with a Kabuki syndrome phenotype demonstrate DNA methylation abnormalities. Eur. J. Hum. Genet. 25, 1335-1344

46. Willemsen, M.H. et al. (2012) Update on Kleefstra syndrome. Mol. Syndromol. 2, 202-212

47. Kleefstra, T. et al. (2006) Loss-of-function mutations in euchromatin histone methyl transferase 1 (EHMT1) cause the $9 \mathrm{q} 34$ subtelomeric deletion syndrome. Am. J. Hum. Genet. 79, 370377

48. Yatsenko, S.A. et al. (2009) Molecular mechanisms for subtelomeric rearrangements associated with the 9q34.3 microdeletion syndrome. Hum. Mol. Genet. 18, 1924-1936

49. Tachibana, M. et al. (2005) Histone methyltransferases G9a and GLP form heteromeric complexes and are both crucial for methylation of euchromatin at H3-K9. Genes Dev. 19, 815-826

50. Liu, N. et al. (2015) Recognition of H3K9 methylation by GLP is required for efficient establishment of $\mathrm{H} 3 \mathrm{~K} 9$ methylation, rapid target gene repression, and mouse viability. Genes Dev. 29, 379-393

51. Jones, W.D. et al. (2012) De novo mutations in MLL cause Wiedemann-Steiner syndrome. Am. J. Hum. Genet. 91, 358-364

52. Milne, T.A. et al. (2002) MLL targets SET domain methyltransferase activity to Hox gene promoters. Mol. Cell 10, 1107-1117

53. Stellacci, E. et al. (2016) Congenital immunodeficiency in an individual with Wiedemann-Steiner syndrome due to a novel missense mutation in KMT2A. Am. J. Med. Genet. A 170, 2389-2393

54. Bogaert, D.J. et al. (2017) Early-onset primary antibody deficiency resembling common variable immunodeficiency challenges the diagnosis of Wiedeman-Steiner and Roifman syndromes. Sci. Rep. 7, 3702

55. Elizondo, L.I. et al. (2009) Schimke immuno-osseous dysplasia: SMARCAL1 loss-of-function and phenotypic correlation. J. Med. Genet. 46, 49-59

56. Boerkoel, C.F. et al. (2000) Manifestations and treatment of Schimke immuno-osseous dysplasia: 14 new cases and a review of the literature. Eur. J. Pediatr. 159, 1-7

57. Boerkoel, C.F. et al. (2002) Mutant chromatin remodeling protein SMARCAL1 causes Schimke immuno-osseous dysplasia. Nat. Genet. 30, 215-220

58. Bansbach, C.E. et al. (2009) The annealing helicase SMARCAL1 maintains genome integrity at stalled replication forks. Genes Dev. 23, 2405-2414

59. Sanyal, M. et al. (2015) Lack of IL7Ralpha expression in T cells is a hallmark of T-cell immunodeficiency in Schimke immunoosseous dysplasia (SIOD). Clin. Immunol. 161, 355-365

60. Baradaran-Heravi, A et al. (2012) Penetrance of biallelic SMARCAL1 mutations is associated with environmental and genetic disturbances of gene expression. Hum. Mol. Genet. 21, 2572-2587

61. Kracker, S. et al. (2015) An inherited immunoglobulin class switch recombination deficiency associated with a defect in the INO80 chromatin remodeling complex. J. Allergy Clin. Immunol. 135, 998-1007

62. Poli, J. et al. (2017) The INO80 remodeller in transcription, replication and repair. Philos. Trans. R. Soc. Lond. B Biol. Sci. 372, 20160290

63. Schulz, Y. et al. (2014) CHARGE and Kabuki syndromes: a phenotypic and molecular link. Hum. Mol. Genet. 23, 4396-4405

64. Basson, M.A. and van Ravenswaaij-Arts, C. (2015) Functional insights into chromatin remodelling from studies on CHARGE syndrome. Trends Genet. 31, 600-611

65. Vissers, L.E. et al. (2004) Mutations in a new member of the chromodomain gene family cause CHARGE syndrome. Nat. Genet. 36, 955-957

66. Wong, M.T. et al. (2015) Immune dysfunction in children with CHARGE syndrome: a cross-sectional study. PLOS One 10, e0142350

67. Roifman, C.M. (1999) Antibody deficiency, growth retardation, spondyloepiphyseal dysplasia and retinal dystrophy: a novel syndrome. Clin. Genet. 55, 103-109

68. Merico, D. et al. (2015) Compound heterozygous mutations in the noncoding RNU4ATAC cause Roifman Syndrome by disrupting minor intron splicing. Nat. Commun. 6, 8718

69. Heremans, J. et al. (2018) Abnormal differentiation of B cells and megakaryocytes in patients with Roifman syndrome. J. Allergy Clin. Immunol. 142, 630-646

70. Craxton, A. et al. (2005) BAFF regulates B cell survival by downregulating the $\mathrm{BH}$-only family member Bim via the ERK pathway. J. Exp. Med. 202, 1363-1374

71. Massaad, M.J. et al. (2013) Wiskott-Aldrich syndrome: a comprehensive review. Ann. N. Y. Acad. Sci. 1285, 26-43

72. Wada, T. et al. (2005) Analysis of T-cell repertoire diversity in Wiskott-Aldrich syndrome. Blood 106, 3895-3897

73. Taylor, M.D. et al. (2010) Nuclear role of WASp in the pathogenesis of dysregulated TH1 immunity in human Wiskott-Aldrich syndrome. Sci. Transl. Med. 2, 37ra44

74. Szabo, S.J. et al. (2000) A novel transcription factor, T-bet, directs Th1 lineage commitment. Cell 100, 655-669

75. Kallies, A. and Good-Jacobson, K.L. (2017) Transcription factor T-bet orchestrates lineage development and function in the immune system. Trends Immunol. 38, 287-297

76. Muramatsu, M. et al. (2000) Class switch recombination and hypermutation require activation-induced cytidine 
deaminase (AID), a potential RNA editing enzyme. Cell 102, 553-563

77. Petersen-Mahrt, S.K. et al. (2002) AID mutates E. coli suggesting a DNA deamination mechanism for antibody diversification. Nature 418, 99-103

78. Revy, P. et al. (2000) Activation-induced cytidine deaminase (AID) deficiency causes the autosomal recessive form of the Hyper-IgM syndrome (HIGM2). Cell 102, 565-575

79. Morgan, H.D. et al. (2004) Activation-induced cytidine deaminase deaminates 5-methylcytosine in DNA and is expressed in pluripotent tissues: implications for epigenetic reprogramming. J. Biol. Chem. 279, 52353-52360

80. Ramiro, A.R. and Barreto, V.M. (2015) Activation-induced cytidine deaminase and active DNA demethylation. Trends Biochem. Sci. 40, 172-181

81. Kunimoto, H. et al. (2017) Aid is a key regulator of myeloid/ erythroid differentiation and DNA methylation in hematopoietic stem/progenitor cells. Blood 129, 1779-1790

82. Milagre, I. et al. (2017) Gender differences in global but not targeted demethylation in iPSC reprogramming. Cell Rep. 18, 1079-1089

83. Teater, M. et al. (2018) AICDA drives epigenetic heterogeneity and accelerates germinal center-derived lymphomagenesis. Nat. Commun. 9, 222

84. Sandoval, J. and Esteller, M. (2012) Cancer epigenomics: beyond genomics. Curr. Opin. Genet. Dev. 22, 50-55

85. Murtha, M. and Esteller, M. (2016) Extraordinary cancer epigenomics: thinking outside the classical coding and promoter box. Trends Cancer 2, 572-584

86. Bartlett, T.E. et al. (2016) Epigenetic reprogramming of fallopian tube fimbriae in BRCA mutation carriers defines early ovarian cancer evolution. Nat. Commun. 7, 11620

87. Fang, D. et al. (2016) The histone H3.3K36M mutation reprograms the epigenome of chondroblastomas. Science 352, $1344-1348$

88. Mack, S.C. et al. (2014) Epigenomic alterations define lethal CIMP-positive ependymomas of infancy. Nature 506, 445-450

89. Sanders, M.A. et al. (2018) MBD4 guards against methylation damage and germ line deficiency predisposes to clonal hematopoiesis and early-onset AML. Blood 132, 1526-1534

90. Kienzler, A.K. et al. (2017) The role of genomics in common variable immunodeficiency disorders. Clin. Exp. Immunol. 188, 326-332

91. de Valles-lbanez, G. et al. (2018) Evaluating the genetics of common variable immunodeficiency: monogenetic model and beyond. Front. Immunol. 9, 636

92. Stray-Pedersen, A. et al. (2017) Primary immunodeficiency diseases: genomic approaches delineate heterogeneous Mendelian disorders. J. Allergy Clin. Immunol. 139, 232-245

93. Abolhassani, H. et al. (2018) Clinical implications of systematic phenotyping and exome sequencing in patients with primary antibody deficiency. Genet Med. Published online June 19, 2018. http://dx.doi.org/10.1038/s41436-018-0012-x

94. Maffucci, P. et al. (2016) Genetic diagnosis using whole exome sequencing in common variable immunodeficiency. Front. Immunol. 7, 220
95. Orange, J.S. et al. (2011) Genome-wide association identifies diverse causes of common variable immunodeficiency. J. Allergy Clin. Immunol. 127, 1360-1367

96. van Schouwenburg, P.A. et al. (2015) Application of whole genome and RNA sequencing to investigate the genomic landscape of common variable immunodeficiency disorders. Clin. Immunol. 160, 301-314

97. Rodriguez-Cortez, V.C. et al. (2015) Monozygotic twins discordant for common variable immunodeficiency reveal impaired DNA demethylation during naive-to-memory B-cell transition. Nat. Commun. 6, 7335

98. Cullen, S.M. et al. (2014) Hematopoietic stem cell development: an epigenetic journey. Curr. Top. Dev. Biol. 107, 39-75

99. Stein, R.A. (2012) Epigenetics and environmental exposures. J. Epidemiol. Community Health 66, 8-13

100. Hedrich, C.M. and Tsokos, G.C. (2011) Epigenetic mechanisms in systemic lupus erythematosus and other autoimmune diseases. Trends Mol. Med. 17, 714-724

101. Hu, D. and Shilatifard, A. (2016) Epigenetics of hematopoiesis and hematological malignancies. Genes Dev. 30, 2021-2041

102. Wu, H. et al. (2018) Epigenetic regulation in B-cell maturation and its dysregulation in autoimmunity. Cell. Mol. Immunol. 15, 676-684

103. Espinoza, J.L. et al. (2018) Infection complications in hematopoietic stem cells transplant recipients: do genetics really matter? Front. Microbiol. 9, 2317

104. Zeiser, R. and Blazar, B.R. (2017) Acute graft-versus-host disease - biologic process, prevention, and therapy. N. Engl. $J$ Med. 377, 2167-2179

105. Deidda, G. et al. (2015) Reversing excitatory GABAAR signaling restores synaptic plasticity and memory in a mouse model of Down syndrome. Nat. Med. 21, 318-326

106. Kuehn, B.M. (2016) Treating trisomies: prenatal Down's syndrome therapies explored in mice. Nat. Med. 22, 6-7

107. Thakore, P.I. et al. (2016) Editing the epigenome: technologies for programmable transcription and epigenetic modulation. Nat. Methods 13, 127-137

108. Balakrishnan, L. and Milavetz, B. (2017) Epigenetic regulation of viral biological processes. Viruses 9, E346

109. Pirrotta, V. (2016) The necessity of chromatin: a view in per spective. Cold Spring Harb. Perspect. Biol. 8, a019547

110. Allis, C.D. and Jenuwein, T. (2016) The molecular hallmarks of epigenetic control. Nat. Rev. Genet. 17, 487-500

111. Zhao, Y. and Garcia, B.A. (2015) Comprehensive catalog of currently documented histone modifications. Cold Spring Harb. Perspect. Biol. 7, a025064

112. Simo-Riudalbas, L. and Esteller, M. (2014) Cancer genomics identifies disrupted epigenetic genes. Hum. Genet. 133, $713-$ 725

113. Berdasco, M. and Esteller, M. (2013) Genetic syndromes caused by mutations in epigenetic genes. Hum. Genet. 132 359-383

114. Brookes, E and Shi, Y (2014) Diverse epigenetic mechanisms of human disease. Annu. Rev. Genet. 48, 237-268

115. Zoghbi, H.Y. and Beaudet, A.L. (2016) Epigenetics and human disease. Cold Spring Harb. Perspect. Biol. 8, a019497 